\title{
Introduction
}

\section{Identifying and validating causal genetic alterations in human breast cancer}

\author{
Stephen P. Ethier \\ Department of Radiation Oncology, Division of Radiation and Cancer Biology, The University of Michigan Health \\ System, and the University of Michigan Comprehensive Cancer Center, Ann Arbor, MI, USA
}

Key words: breast cancer oncogene, gene amplification

\begin{abstract}
Summary
An important mechanism for the activation of proto-oncogenes in human breast and other cancers is gene amplification, which results in gene overexpression at both the message and the protein levels. Recent studies have demonstrated that oncogenes rarely if ever become amplified in isolation, but rather are present on large amplicons that contain multiple genes. More detailed analysis of these amplicons has revealed the presence of many candidate breast cancer oncogenes. The broad goal of this issue of Breast Cancer Research and Treatment is to review the current state of our understanding of the causal role of defined genetic alterations that occur in human breast cancers, and to discuss the case for the mechanistic significance of several candidate oncogenes. As will be seen, these studies have revealed a remarkable genetic complexity and heterogeneity in human breast cancer that must be dissected in order to improve our mechanistic understanding of disease progression, and to develop effective new drugs against relevant molecular targets.
\end{abstract}

\section{Defining causal genetic alterations in human breast cancer}

This special edition of Breast Cancer Research and Treatment is devoted to reviewing the state of our understanding of the causal genetic alterations that underlie the majority of human breast cancers. It is important that the true genetic causes of human breast cancer be defined in order to clearly understand the mechanistic basis of breast cancer progression. In addition, it is now clear from the early clinical trials of targeted therapeutics, that the products of genes that are most directly linked to the expression of neoplastic phenotypes provide the best targets for new drug development.

As often happens in science, advances in our knowledge on the genetics of human breast cancer has served to cloud, more than it has served to clarify, our understanding of the genetic basis of breast cancer. This, in itself, serves as an important reminder of just how little we understand about human breast cancer genetics. For example, just a few years ago, one could have generated a simple list of likely human breast cancer genes, which would have included HER-2, C-MYC, FGFR1, and perhaps some of the int genes, such as FGF-3 and FGF-4 [1]. Further study of each of these genes and their respective genetic loci have demonstrated that with the exception of HER-2, the genes listed above may serve as little more than genetic markers for other more mechanistically relevant genes that are present in the same regions of the genome. This topic will be discussed in great detail throughout this volume.

It can be argued that the only known and validated human breast cancer oncogene is HER-2. It is the only gene that has been strongly associated with human breast cancer progression in studies of clinical material $[2,3]$ that has also been shown to be transforming in human mammary epithelial cells in vitro [4, 5], to cause mammary tumor development in transgenic mice $[6,7]$, and for which a targeted therapeutic has been developed that has efficacy in the clinic $[8,9]$. Thus, the case for HER-2 as a human breast cancer oncogene is strong and is based on both correlative 
and mechanistic data. However, even for HER-2, the mechanism by which the gene becomes activated as an oncogene in human breast and other cancers has demonstrated that the picture is more complicated than would appear based on the evidence described above. Thus, HER-2, like other candidate oncogenes, becomes activated by a process of gene amplification, which results in large increases in copy number of the gene, and which is accompanied by large increases in message and protein levels. However, HER-2 does not undergo gene amplification in isolation. Rather, HER2 is part of a large amplicon centered around chromosome $17 \mathrm{q} 12$ that contains many amplified genes in addition to HER-2. And, as will be discussed in detail in the chapter by Jarvinen, some of the genes on the amplicon may have mechanistic significance and act either in concert with, or in addition to, the transforming effects of HER-2. In addition, the HER2 story has served to illustrate the important scientific principle that correlation does not prove causation in an area that has direct clinical relevance. Again, as described in detail by Jarvinen, correlative clinical data have suggested a link between HER-2 amplification and response to adriamycin containing chemotherapy regimens. Despite this correlation, mechanistic data from the laboratory to support that finding have not been found. More recently, however, it has become clear that another gene that is sometimes present on the HER-2 amplicon, namely Topoisomerase II, is associated with adriamycin responsiveness [10]. The mechanistic connection between TOPO II overexpression and response to adriamycin has been demonstrated in the laboratory. Thus, studies of the HER-2 gene, and more importantly, the HER-2 amplicon, have served to illuminate an important principle of human cancer genetics; that focal areas of gene amplification mark the presence of dominantly acting oncogenes, and that multiple genes on an amplicon may have a role in breast cancer progression.

The majority of chapters in this volume are dedicated to reviewing the state of our knowledge regarding the most common amplicons in human breast cancer, and what we currently understand about the candidate oncogenes present at those loci. As with the HER-2 locus on chromosome 17q12, the other common amplicons contain several genes that could, in certain contexts, act as breast cancer oncogenes. Donna Albertson will discuss the current state-of-theart in defining regions of gene amplification in breast cancers. As indicated above, Jarvinen and Liu will discuss the HER-2 amplicon, and Sinclair et al. will describe a second amplicon on chromosome 17q21, which also contains several interesting breast cancer oncogenes. The chapter by Hodgson et al. details the amplicon on chromosome 20q12-q13 that contains at least three genes for which there is mechanistic evidence for a role in breast cancer progression. The chapter by Ormandy et al. will outline a particularly interesting amplicon in breast cancer that centers around chromosome 11q13. This amplicon has seen the emergence and disappearance of a number of candidate genes over the years. The current view is that at least two genes on that amplicon, CCND1 and EMS1, are likely to play mechanistic roles in human breast cancer. The unifying theme for all of these chapters is that, without exception, each amplicon that has been identified in human breast cancer contains more than one candidate oncogene for which there is evidence for a causal role in breast cancer development.

In the final chapter, Peter O'Connell will summarize the state of our knowledge of an even more complicated area of breast cancer genetics: the role of loss of genetic material at specific loci as indicators of putative tumor suppressor genes. This is an even more difficult area of work because, unlike amplicons where the search for oncogenes can be narrowed by focusing on the subset of genes that are overexpressed, any and all genes on chromosomal regions commonly lost in breast cancer are potential candidate tumor suppressor genes.

\section{Mechanistic implications of focal areas of gene amplification}

Given our current understanding of the candidate breast cancer genes present on focal amplicons, how do we interpret the fact of their existence in breast and other human cancers? The prevailing view just a few years ago was that each amplicon harbored a single oncogene. However, based on our current understanding of the plethora of candidate genes that have been identified in a small number of amplicons, we must now begin to consider an alternate view. Thus, it may be that focal areas of gene amplification emerge in human cancers non-randomly because certain areas of the genome harbor multiple protooncogenes in relatively close proximity. Furthermore, it may be possible that multiple proto-oncogenes on a single amplicon may interact mechanistically to drive tumor progression. Indeed, it is not difficult to hypothesize that the simultaneous overexpression of HER-2 
and Grb-7, which are commonly co-overexpressed from the same amplicon, cooperate in the expression of transformed phenotypes. Grb7 has been recently linked to signaling via focal adhesion kinase (FAK), which plays an important role in motility and invasion [11-13]. We demonstrated that when HER-2 is overexpressed, it interacts directly with FAK, and that HER-2 overexpression yields a motile and invasive phenotype [5, 14]. Thus, co-overexpression of HER-2 and Grb7 may be mechanistically relevant and may be illustrative of the importance of amplicons containing multiple genes that act together in cancer progression. Focal areas of gene amplification may, therefore, be an efficient mechanism for cells to activate multiple oncogenes that cooperate to transform cells.

\section{Breast cancer genes in tumors without focal areas of gene amplification}

Clearly, focal areas of gene amplification provide important areas of the genome in which to search for breast cancer genes. However, it is important to keep in mind that not all breast cancers exhibit defined amplicons. Indeed, Albertson and colleagues have recently estimated that only a fraction of all breast cancers have these kinds of genetic alterations. The remaining breast cancers either have low level chromosomal changes in the majority of chromosomes, or have structurally normal chromosomes, even though they may be aneuploid. Thus, even after we have identified all of the causally relevant oncogenes present on focal amplicons, there will still be an enormous challenge to understand the genetic basis of breast cancer progression in the large fraction of tumors that remain. As alluded to above, these studies are critically important to the development of novel targeted therapeutic agents. While correlative studies of genes and clinical outcome may be sufficient for studies of prognosis and for the identification of biomarkers, the development of new therapies for breast cancer patients absolutely depends on a mechanistic understanding of the genetic basis of human breast cancer progression. The principle that effective drugs against good targets yields great clinical benefit has been proven with drugs such as tamoxifen, herceptin (Trastuzumab) and Gleevec. However, the clinical lessons learned so far with these agents is that, despite their target specificity and effectiveness, more such agents directed against other mechanistic targets must be developed so that remissions can be converted into cures for patients with breast and other cancers.

\section{References}

1. Ethier SP: Growth factor synthesis and human breast cancer progression. J Natl Cancer Inst 87: 964-973, 1995

2. Slamon DJ, Clark GM, Wong SG, Levin WJ, Ullrich A, McGuire WL: Human breast cancer: correlation of relapse and survival with amplification of the HER-2/neu oncogene. Science 235: 177-182, 1987

3. Borg A, Tandon AK, Sigurdsson H, Clark GM, Ferno M, Fuqua SAW, Killander D, McGuire WL: HER-2/neu amplification predicts poor survival in node-positive breast cancer. Cancer Res 50: 4332-4337, 1990

4. Woods Ignatoski KM, LaPointe AJ, Radany EH, Ethier SP: ErbB-2 overexpression in human mammary epithelial cells confers growth factor independence. Endocrinology 140: 3615-3622, 1999

5. Woods Ignatoski KM, Maehama T, Markwart SM, Dixon JE, Livant DL, Ethier SP: ErbB-2 overexpression confers PI $3^{\prime}$-kinase-dependent invasion capacity on human mammary epithelial cells. Br J Cancer 82: 666-674, 2000

6. Muller WJ, Sinn E, Pattengale PK, Wallace R, Leder P: Singlestep induction of mammary adenocarcinoma in transgenic mice bearing the activated c-neu oncogene. Cell 54: 105-115, 1988

7. Siegel PM, Dankort DL, Hardy WR, Muller WJ: Novel activating mutations in the neu proto-oncogene involved in induction of mammary tumors. Mol Cell Biol 14: 7068-7077, 1994

8. Leyland-Jones B, Smith I: Role of herceptin (r) in primary breast cancer: views from North America and Europe. Oncology 61: 83-91, 2001

9. Spiridon CI, Ghetie MA, Uhr J, Marches R, Li JL, Shen GL, Vitetta ES: Targeting multiple Her-2 epitopes with monoclonal antibodies results in improved antigrowth activity of a human breast cancer cell line in vitro and in vivo. Clinical Cancer Res 8: 1720-1730, 2002

10. Jarvinen TA, Tanner M, Rantanen V, Barlund M, Borg A, Grenman S, Isola J: Amplification and deletion of topoisomerase II alpha associate with ErbB-2 amplification and affect sensitivity to topoisomerase II inhibitor doxorubicin in breast cancer. Am J Pathol 156: 839-847, 2000

11. Fiddes RJ, Campbell DH, Janes PW, Sivertsen SP, Sasaki H, Wallasch C, Daly RJ: Analysis of Grb7 recruitment by heregulin-activated ErbB receptors reveals a novel target selectivity for ErbB3. J Biol Chem 273: 7717-7724, 1998

12. Han DC, Shen TL, Guan JL: Role of Grb7 targeting to focal contacts and its phosphorylation by focal adhesion kinase in regulation of cell migration. J Biol Chem 275: 28911-28917, 2000

13. Tanaka S, Sugimachi K, Kawaguchi H, Saeki H, Ohno S, Wands JR: Grb7 signal transduction protein mediates metastatic progression of esophageal carcinoma. J Cell Physiol 183: 411-415, 2000

14. Woods Ignatoski K, Ethier SP: Constitutive activation of pp 125 fak in eleven newly isolated breast cancer cell lines. Breast Cancer Res Treatment 54: 173-182, 1999

Address for offprints and correspondence: Stephen P. Ethier, Department of Radiation Oncology, Division of Radiation and Cancer Biology, The University of Michigan Health System, and the University of Michigan Comprehensive Cancer Center, 1500 E. Medical Center Dr., Ann Arbor, MI 48109-0982, USA; Tel.: +1-734-647-1008; Fax: +1-734-647-9480; E-mail: spethier@umich.edu 